\title{
Enhancements to Online Help: Adaptivity and Embodied Conversational Agents
}

\author{
Jérôme Simonin and Noëlle Carbonell \\ INRIA (Nancy Grand-Est Research Center), Henri Poincaré University \\ LORIA, Campus Scientifique \\ BP 70239, Vandoeuvre-lès-Nancy Cedex, France \\ \{Jerome.Simonin, Noelle.Carbonell\}@loria.fr
}

\begin{abstract}
We present and discuss the results of two empirical studies that aim at assessing the contributions, to the effectiveness and efficiency of online help of: adaptive-proactive user support (APH), multimodal (speech and graphics) messages (MH), and embodied conversational agents (ECAs). These three enhancements to online help were implemented using the Wizard of $\mathrm{Oz}$ technique. The first study (E1) compares $\mathrm{MH}$ with APH, while the second study (E2) compares MH with embodied help (EH). Half of the participants in E1 (8) used $\mathrm{MH}$, and the other half used $\mathrm{APH}$. Most participants who used $\mathrm{MH}$, resp. $\mathrm{APH}$, preferred $\mathrm{MH}$, resp. APH, to standard help systems which implement text and graphics messages (like APH). In particular, proactive assistance was much appreciated. However, higher performances were achieved with MH. A majority of the 22 participants in E2 preferred $\mathrm{EH}$ to $\mathrm{MH}$, and were of the opinion that the presence of an ECA, a talking head in this particular case, has the potential to improve help effectiveness and efficiency by increasing novice users' self confidence. However, performances with the two systems were similar, save for help consultation rate which was higher with EH. Longitudinal (usage) studies are needed to confirm the effects of these three enhancements on novice users' judgments and performances.
\end{abstract}

Keywords: Adaptive user interfaces, Embodied conversational agents, Talking heads, Online help, Speech and graphics, Multimodal interaction, Eye tracking.

\section{Introduction}

The effectiveness of online help for the general public is still unsatisfactory despite continuous efforts from researchers and designers over the last twenty years. Help facilities are still ignored by most "lay users" who prefer consulting experienced users to browsing online manuals. This behavior is best accounted for by the "motivational paradox" [4], that is: users in the general public are reluctant to explore the functionalities of unfamiliar software and learn how to use them efficiently, as their main objective is to carry out the tasks they have in mind. The two studies reported here address the crucial issue of how to design online help that will actually be used by the general public, an essential condition for ensuring its effectiveness. 
To achieve this goal, help systems should be capable of providing users with appropriate information right when they need it; according to the "minimum manual" [4]. They should be aware of the user's current goal, knowledge and skills to meet both requirements; that is, they should have the capability to create and update an adaptive model of the current user's profile from interaction logs analysis. Such a model is needed for (i) tailoring help information to the user's current knowledge, and (ii) anticipating their information needs accurately so as to satisfy them through timely initiatives. This help strategy, which is both adaptive and proactive, is liable to improve help effectiveness and alleviate users' cognitive workload.

Using speech for conveying user support information may also contribute to increase online help usage, hence its effectiveness, by reducing the interference of help consultation in the user's main activity: users have to stop interacting with software applications to read textual help information which is usually superimposed on the application window. In contrast, oral messages do not use screen space, and users can easily go on interacting with the application while listening to oral messages; in particular, they can carry out a sequence of oral instructions while it is being delivered.

Finally, novice users' motivation and emotional state strongly influence the effecttiveness of knowledge or skill acquisition. Embodied online help has the potential to encourage requests for assistance; it may also increase novice users' self-confidence and reduce their stress.

We present two empirical studies, E1 and E2, which attempt to assess the actual contributions of three possible enhancements to online help effectiveness and userfriendliness: adaptivity and proactivity, speech- and graphics-based messages, and embodied conversational agents (ECAs). The aim is to determine whether these enhancements have the potential to win user acceptance and improve novice users' performances, especially memorization of the procedural and semantic knowledge needed to operate standard applications effectively. The method is described in the next section. Results are presented and discussed in the third section.

\section{Related Work}

Adaptive online help has motivated large-scale research efforts, such as the Lumiere project [8] or the Berkeley Unix Consultant [23]. According to [5] and [9], the bulk of research on adaptive human-computer interaction has been focused on designing efficient user models. Contrastingly, assessment of these models and evaluation of the effectiveness and usability of adaptive user interfaces are research areas which need to be further developed.

Evaluation of the ergonomic quality of speech as an output modality has motivated few studies compared to speech input. Recent research has been centered on issues pertaining to the use of speech synthesis in contexts where displays are difficult to use; see, for instance, interaction with in-vehicle systems [24] and mobile devices [19], or interfaces for sight impaired users [18]. Speech synthesis intelligibility [1] and expressiveness (especially the use of prosody for conveying emotions [22] have also motivated a number of studies. In contrast, to our best knowledge, the use of speech for expressing help information has been investigated by only one research 
group. Authors of [10] propose guidelines for the design and test of help information presented via voice synthesis to users of current commercial computer applications.

ECAs with humanlike appearance and advanced verbal and nonverbal communication capabilities have been designed and implemented in many research laboratories. Current research efforts focus on creating ECAs which emulate, as best as possible, human appearance, facial expressions, gestures and movements, emotions and intelligent behaviors. Modeling expression of emotions [17], human conversational skills [16] and gaze activity during face-to-face exchanges [7] are very active research areas. Most evaluation studies of ECAs' contributions to human-computer interaction focus on computer-aided learning situations; for a survey, see [14]. For instance, [12] investigates students' motivations for acceptance or rejection of ECAs. In other application domains, utility and usability of humanlike ECAs have motivated only a few ergonomic studies ${ }^{1}$. Save for the pioneer work reported in [21], most evaluation studies address only a few issues in the design space for humanlike ECAs, such as the influence of the ECA's voice quality (extrovert vs introvert) on children performances [6], or the affective dimension of interaction with a humanlike ECA (7 out of the 9 studies mentioned in [20].

\section{Method}

\subsection{Overview}

We used the same methodology and setup for E1 and E2, in order to compare the effects of the three proposed enhancements to online help on learning how to operate an unfamiliar software application. We chose Flash, a software tool for creating graphical animations, because computer-aided design of animations involves concepts which differ from those implemented in standard interactive software for the general public. Thus, participants in E1 and E2, who were unfamiliar with animation creation tools, had to acquire both semantic knowledge and procedural know-how in order to carry out the proposed animation creation tasks using Flash.

Undergraduate students (16 for E1, 22 for E2) who had never used Flash or any other animation tool, had to create two animations. E1 participants were divided into two gender-balanced groups with 8 participants each; one group could consult an adaptive-proactive help system (APH), and the other group a multimodal system (MH). Two online help systems, the MH system and an embodied help system (EH), were put successively at the disposal of E2 participants, one per animation; presentation order of $\mathrm{MH}$ and $\mathrm{EH}$ was counterbalanced among participants. Withinparticipant design, which reduces the effects of inter-individual differences, was not adopted for E1 to ensure that participants would notice message content evolutions. The MH system (used in E1 and E2) delivered oral help information illustrated with copies of Flash displays. The same database of multimodal help messages (over 300 messages) was used for $\mathrm{MH}$ and $\mathrm{EH}$. EH was embodied by a female talking head which "spoke" speech components of messages. Speech was transcribed into printed text for APH so as to avoid implementing two enhancements in the same system.

\footnotetext{
${ }^{1}$ e.g., Only 1 session (out of 8) was devoted to evaluation at IVA'07.
} 
Once participants had filled out a background information questionnaire (10 $\mathrm{min}$.), they got acquainted with Flash basic concepts (e.g., 'scenario', 'interpolation', etc.) using a short multimedia tutorial they could browse through as long as they wanted to (15-20 min.). Then, they tried to reproduce two predefined animations (1 hour 15 min. or so). During the second study only, their gaze movements were recorded throughout their interactions with Flash and the help systems, so as to obtain objective data on participants' subjective reactions to the ECA's presence. Afterwards, they filled out two questionnaires, a verbal and a nonverbal one [2]; both questionnaires were meant to elicit their subjective judgments on the help system(s) they had experimented; Lickert scales and Osgood's semantic differentiators were preferably used for collecting verbal judgments. Next, their understanding and memorization of Flash basic concepts and procedures were assessed using a written post-test. Finally, they participated in a debriefing interview. All-in, individual sessions lasted about two hours and a half.

\subsection{Implementation of the Three Help Systems}

To reduce interference between help consulting and animation design, the display included two permanent windows (see figure 1), a sizeable Flash window and a small help window (on the right of the screen).

Based on earlier empirical work [3], participants could request four different types of help information using four dedicated buttons: procedural know-how (How?), semantic knowledge (What?), explanations of the application current state (Why?) and confirmation or invalidation of most recent Flash commands (Confirm?). Oral help messages were activated using colored buttons placed above Flash display copies illustrating their content. Speech acts were indicated by colors: warning (e.g., precondition), concept definition or procedure description, and explanations or recommendations. Colors were also used to denote speech acts in APH textual messages. The talking head was placed at the top of the EH window.

To achieve realistic adaptive-proactive assistance, we resorted to the Wizard of $\mathrm{Oz}$ paradigm as a rapid prototyping technique. We also used it for $\mathrm{EH}$ and $\mathrm{MH}$ so as to ensure identical reaction times to participants' requests for the three simulated systems. The Wizard was given software facilities to adapt the information content of help messages to the current participant's actual knowledge and skills as s/he perceived them through observing their interactions with Flash and the help system. Three different versions of each message (M) were available to the Wizard:

- An initial version (V1) including all the semantic and procedural information needed by users unfamiliar with the information in $\mathrm{M}$; the Wizard used it to answer participants' first request for $\mathrm{M}$.

- To answer further requests for M, s/he had to choose between two other versions:

a short reminder of the information in V1, with or without additional technical details; s/he activated it for participants who had shown a good understanding of the information in V1 during interactions with Flash subsequent to their first request for $\mathrm{M}$;

a detailed presentation of the information in V1, including explanations, examples and/or illustrations; it was intended for participants who had experi-enced difficulties in understanding and putting to use the information in V1. 


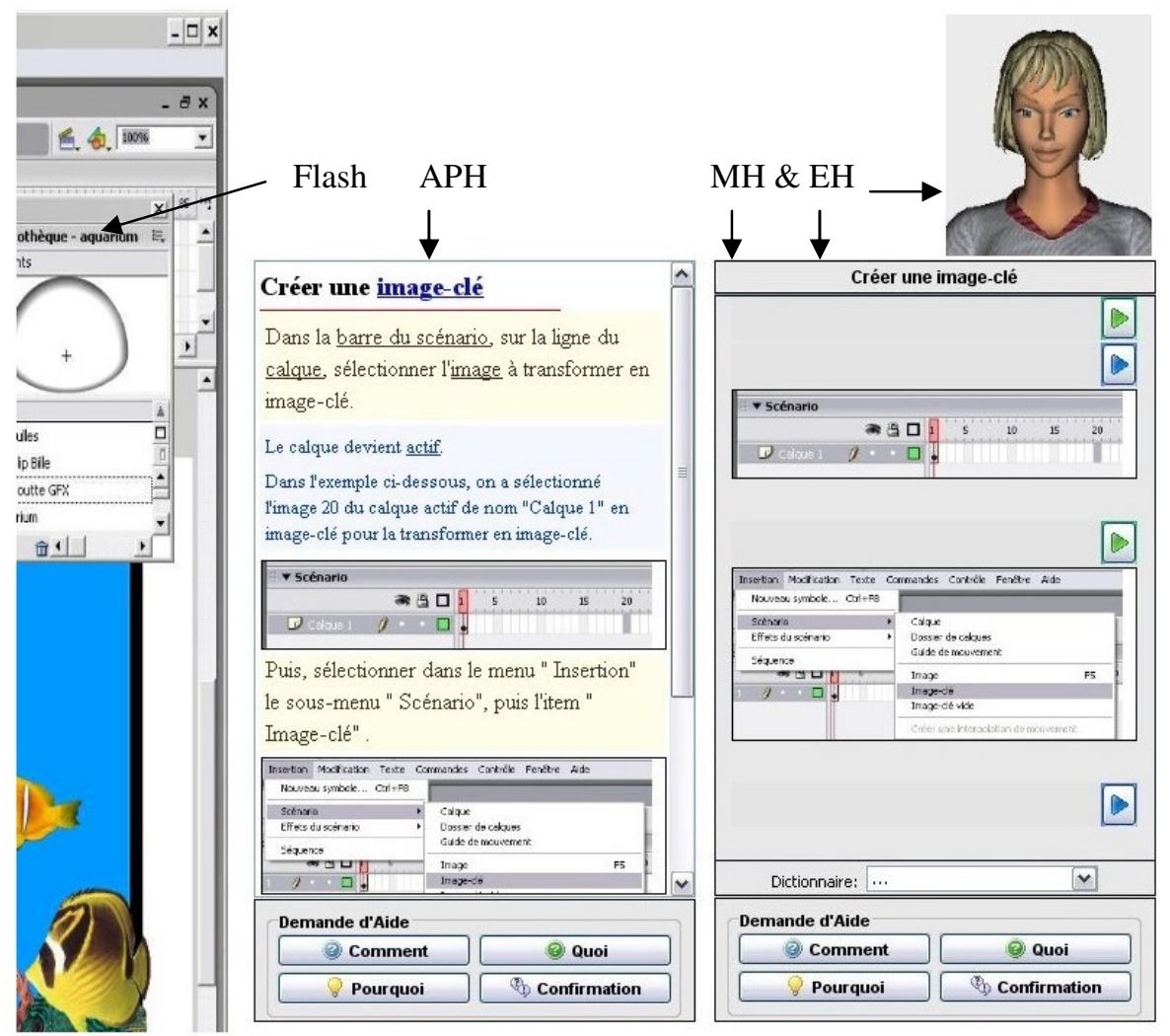

Fig. 1. The three help systems, APH in the center, MH/EH on the right, and Flash window on the left

Practically, when a participant sent a request to the APH system, a software assistance platform displayed the name of the appropriate message on the Wizard's screen. Thus, the Wizard had just to select the version of the message that best matched this participant's current knowledge and skills, based on the observation of her/his interactions with Flash; the message was automatically displayed on the participant's screen. As participants' interactions with Flash and the APH system lasted one hour at the most, three versions of the same message were sufficient to achieve realistic simulations of dynamic adaptation to the evolution of participants' familiarity with Flash through the session. When simulating $\mathrm{MH}$ or $\mathrm{EH}$, the Wizard had just to activate the message selected by the assistance platform.

To implement proactive user support, the Wizard was instructed to observe participants' interactions with Flash, and assist them in creating the two predefined animations by anticipating their information needs and satisfying them on her/his own initiative using appropriate versions of messages in the database. 


\subsection{Measures}

Post-session questionnaires and debriefings were analyzed in order to gain an insight into participants' subjective judgments on the help system(s) they had experimented.

To assess the influence of each enhancement to online help on participants' performances and behaviors, we used post-test marks and analyses of manually annotated interaction logs. These data provided us with information on participants' assimilation of Flash concepts and operation, help usage and task achievement. Some analyses of annotated logs were restricted to the first animation creation task which lasted about 40 minutes on average, as participants seldom requested help or needed pro-active assistance while creating the second animation, most of the necessary knowledge to carry it out having been acquired during execution of the first task.

To assess participants' affective responses to the presence of the ECA, behaviorbased measures were collected in addition to verbal and non verbal judgments, as recommended in [15]. E2 participants' gaze movements were recorded (at $60 \mathrm{~Hz}$ ) throughout the session, using a head-mounted eye tracker (ASL-501) which allows free head and chest movements without loss of precision. As voluntary eye movements express shifts of visual attention, they are valuable indicators of users' engagement and involvement in interaction with an ECA [11]. Physiological measures, such as heart rate or galvanic skin response [13] were ignored, as they are more intrusive than eye tracking in standard HCI environments.

\subsection{Software Developments}

We developed a client-server platform to assist the Wizard in his/her task and tools for facilitating annotation and analysis of interaction logs. These software developments in Java are briefly described in this section.

Software Assistance to the Wizard's Activity. The client-server platform can: display copies of the participant's screen on the Wizard's screen; display help messages activated by the Wizard on the participant's screen; assist the Wizard in the simulation of the three help systems by selecting the message, M, matching the participant's current request; and, for the APH system, provide her/him with a history of all versions of $M$ sent to the participant previously. Messages are stored in a hierarchical database as multimedia Web pages. The platform also records logs of participants' interactions with both Flash and the current help system. Time-stamped logs comprise user and system events, mouse positions and clicks, screen copies, eye tracking samples; they may also include recordings of the user's speech and gestures.

Software Assistance to Annotation and Analysis of Interaction Logs. Interaction logs saved by the platform can be "replayed" with gaze points or fixations superimposed on displays. Main annotation facilities include: interactive segmentation and labeling of logs; and, for eye movement analysis, automatic or manual definition of 'areas of interest' over display sequences. Graphical facilities are also provided for visualizing results of eye tracking data analyses (e.g., heat maps). 


\section{Main Results}

\subsection{Participants' Subjective Judgments}

First Study. 6 participants out of 8 preferred the APH system to standard online help. The two remaining participants preferred standard help, due to "the force of habit" according to them. Proactive user support raised enthusiastic comments while message content evolution (adaptivity) went almost unnoticed. 7 participants rated the support provided by APH as very useful, and its initiatives as most effective.

Similarly, 7 participants rated the $\mathrm{MH}$ system higher than standard online help. However, only 5 of them preferred audio to visual presentations of help information, based on the observation that one could carry out oral instructions while listening to them. The 3 other participants who preferred visual presentations explained that taking in spoken information is a more demanding cognitive task than assimilating the content of a text: one can read displayed a textual message at one's pace, and freely select or ignore parts of it, which is impossible with spoken information.

Second Study. According to verbal questionnaires, 16 participants (out of 22) preferred the $\mathrm{EH}$ system to the $\mathrm{MH}$ system. 5 participants rated $\mathrm{MH}$ higher than $\mathrm{EH}$, whereas only one participant gave preference to standard help facilities. Most participants were of the opinion that the presence of an ECA had not disrupted their animation creation activity (17 participants), that it could greatly increase the effectiveness (19), and appeal (21) of online help. Non verbal judgments in Sam $1^{\text {st }}$ line are also very positive: 19 participants enjoyed the ECA's presence, the feelings of the 3 remaining participants being neutral. In addition, 14 participants had the impression that the ECA's presence increased their self-confidence and reduced their stress (Sam $3^{\text {rd }}$ line).

Analyses of annotated eye tracking data during the first task (40 min. or so) indicate that, from the beginning to the end of task execution, all participants (11) glanced at the ECA whether it was talking or silent. Each participant looked at it 75 times on average. Fixation duration was longer while the ECA was talking than when it was silent. These objective measures indicate that the ECA actually succeeded in arousing participants' interest and maintaining it throughout the first task execution, although all of them were primarily intent on achieving the first animation creation task. These results confirm judgments expressed in questionnaires.

\subsection{Participants' Performances and Behaviors}

First Study. Duration of the first task execution greatly varied from one participant to another. Inaction rate, that is, the percentage of time while the mouse remained still, is sensibly higher for the APH group than for the $\mathrm{MH}$ group (62\% versus 53\%). This difference illustrates the efficiency of spoken compared to textual help messages: one has to stop interacting with the application while reading a textual message as mentioned by some participants. APH participants consulted help as often as $\mathrm{MH}$ participants (58 requests vs 60 on the whole), although the APH system displayed 183 messages on its own initiative. Pushing help information to novice users does not seem to reduce the number of help requests. This suggests that proactive assistance is an efficient strategy for increasing help effectiveness, as APH participants read most of the help messages pushed to them, according to debriefings. 
Analysis of post-test marks suggests that $\mathrm{MH}$ participants gained a better understanding of Flash concepts than APH participants and recollected procedures to activate its functions better (average marks: 17.6/31 vs 15.6/31). As for task achievement, the difference between the two groups (MH: 12.6/20 vs APH: 11.1/20 for the first task) may be due to the necessity, for APH participants, to stop interacting with Flash to read help messages. These interruptions may have interfered with task achievement.

Second Study. We divided participants into two groups (of 11 participants each), according to the help system they had used first, $\mathrm{EH}$ or $\mathrm{MH}$. For most measures, average values computed over each group are not sensibly different. This is the case for post-test marks and, concerning the first task, for task execution duration (40 min.), the number of interactions with Flash and the help system, task achievement. The only noticeable difference between the two groups is the average number of help message activations per participant during the first task: 22 for $\mathrm{EH}$ vs 16 for $\mathrm{MH}$. This result suggests that the presence of an ECA may encourage help consultation.

\subsection{Discussion}

According to E1 results, proactive online assistance is likely to arouse higher subjective satisfaction among novice users than adaptive help or multimodal (speech and graphics) messages. Evolutions of message content may have gone unnoticed because adaptivity is a basic feature of human communication, especially in the context of tutor-novice dialogues.

Participants' rather balanced judgments on the usability of speech compared to text should not deter designers from considering speech as a promising alternative modality to text for conveying help information. Speech usability can easily be improved by implementing advanced audio browsing facilities. Taking up this research direction might prove to be more rewarding in the short term than implementing effective proactive help, which still raises unsolved scientific issues. Firstly, to guess novice users' intents accurately from their interactions with an unfamiliar application is a difficult challenge, as these users tend to perform actions unrelated to the achievement of their goals. Secondly, the MH group achieved better performances than the APH group. Efficient proactive help may have induced APH participants to rely on help information to achieve the two animation creation tasks, hence, to put little effort into learning Flash. Additional empirical evidence is needed to validate this interpretation, as the number of participants in E1 was limited.

The presence of an ECA was well accepted by E2 participants. The EH system was preferred to the MH system by most participants who stated that the ECA's presence had not interfered with their animation creation activities. The vast majority of them considered that the presence of an ECA had the potential to improve help effectiveness through increasing users' motivation and self-confidence. However, these perceptions are at variance with their actual performances which were similar for EH and MH. The ECA's presence had no noticeable effect on Flash semantic and procedural knowledge acquisition, task execution time or task achievement; it only encouraged help consultation. Nevertheless, observation of novice users' behaviors and activities over longer time spans may be necessary in order to perceive its possible influence on learning new concepts and skills. Longitudinal studies are 
essential to obtain conclusive evidence on the effects of the presence of an ECA on novice users' performances and its contribution to online help effectiveness and efficiency. Such studies are also needed to determine whether users will not get bored with the presence of an ECA in the long run, and prefer $\mathrm{MH}$ to $\mathrm{EH}$, contrary to E2 results. A few participants commented that the usefulness of the ECA would decrease as the user's knowledge and practice would grow. Performing such studies is a longterm objective as their design and implementation raise many difficult research issues.

\section{General Conclusion}

We have presented and discussed the results of two empirical studies that aim at assessing the contributions, to the effectiveness and efficiency of online help of: adaptive-proactive user support (APH), multimodal (speech and graphics) messages $(\mathrm{MH})$, and embodied conversational agents (ECAs). These three enhancements to online help were implemented using the Wizard of $\mathrm{Oz}$ technique.

The first study (E1) compares MH with APH, while the second study (E2) compares $\mathrm{MH}$ with embodied help (EH). Half of the participants in E1 (8) used $\mathrm{MH}$, and the other half used APH. Most participants who used MH, resp. APH, preferred $\mathrm{MH}$, resp. APH, to standard help systems which implement text and graphics messages. In particular, proactive assistance was much appreciated. However, higher performances were achieved with $\mathrm{MH}$.

A majority of the 22 participants in E2 preferred $\mathrm{EH}$ to $\mathrm{MH}$, and were of the opinion that the presence of an ECA, a talking head in this particular case, has the potential to improve help effectiveness and efficiency by increasing novice users' self confidence. However, performances with the two systems were similar, save for help consultation rate which was higher with $\mathrm{EH}$.

These results open up several research directions. Improving browsing through oral messages is a short-term promising direction. Longitudinal studies are needed to assess whether APH and EH have the potential to improve online help effectiveness and efficiency, especially semantic and procedural knowledge learning. They are also needed to determine whether users will get bored or irritated in the long run by these two enhancements. Design and development of an adaptive-proactive help system, is a long-term research direction, due to the numerous issues that still need to be solved.

\section{References}

1. Axmear, E., Reichle, J., Alamsaputra, M., Kohnert, K., Drager, K., Sellnow, K.: Synthesized Speech Intelligibility in Sentences: a Comparison of Monolingual EnglishSpeaking and Bilingual Children. Language, Speech, and Hearing Services in Schools 36, 244-250 (2005)

2. Bradley, M., Lang, P.: Measuring emotion: the self assessment manikin and the semantic differential. Journal of Behavioral Therapy and Experimental Psychiatry 25, 49-59 (1994)

3. Capobianco, A., Carbonell, N.: Contextual online help: elicitation of human experts' strategies. In: Proc. HCI International 2001. LEA, vol. 2, pp. 824-828 (2001)

4. Carroll, J.M., Smith-Kerber, P.L., Ford, J.R., Mazur-Rimetz, S.A.: The minimal manual. Human-Computer Interaction 3(2), 123-153 (1987) 
5. Chin, D.N.: Empirical Evaluation of User Models and User-Adapted Systems. User Modeling and User-Adapted Interaction 11, 181-194 (2001)

6. Darves, C., Oviatt, S.: Talking to digital fish: Designing effective conversational interfaces for educational software. In: Pélahaud, C., Ruttkay, Z. (eds.) From brows to trust: Evaluating embodied conversational agents. Part IV, ch. 10. Kluwer, Dordrecht (2004)

7. Eichner, T., Prendinger, H., André, E., Ishizuka, M.: Attentive presentation agents. In: Pelachaud, C., Martin, J.-C., André, E., Chollet, G., Karpouzis, K., Pelé, D. (eds.) IVA 2007. LNCS, vol. 4722, pp. 283-295. Springer, Heidelberg (2007)

8. Horvitz, E., Breese, J., Heckerman, D., Hovel, D., Rommelse, K.: The Lumière Project: Bayesian User Modeling for Inferring the Goals and Needs of Software Users. In: Proc. UAI 1998, pp. 256-265 (1998)

9. Jameson, A.: Adaptive Interfaces and Agents. In: Jacko, J., Sears, A. (eds.) HumanComputer Interaction Handbook, ch. 15, pp. 305-330. Erlbaum, Mahwah (2003)

10. Kehoe, A., Pitt, I.: Designing help topics for use with text-to-speech. In: Proc. DC 2006, pp. 157-163. ACM Press, New York (2006)

11. Ma, C., Prendinger, H., Ishizuka, M.: Eye Movement as an Indicator of Users' Involvement with Embodied Interfaces at the Low Level. In: Proc. AISB 2005 Symp. U. of Hartfordshire, pp. 136-143 (2005)

12. Moreno, R., Flowerday, T.: Students' choice of animated pedagogical agents in science learning: A test of the similarity attraction hypothesis on gender and ethnicity. Contemporary Educational Psychology 31, 186-207 (2006)

13. Mori, J., Prendinger, H., Ishizuka, M.: Evaluation of an Embodied Conversational Agent with Affective Behavior. In: Proc. Workshop on Embodied Conversational Characters as Individuals, at AAMAS 2003, pp. 58-61 (2003)

14. Payr, S.: The university's faculty: an overview of educational agents. Applied Artificial Intelligence 17(1), 1-19 (2003)

15. Picard, R.W., Daily, S.B.: Evaluating Affective Interactions: Alternatives to Asking What Users Feel. In: Workshop on Evaluating Affective Interfaces: Innovative Approaches, at CHI 2005 (2005), http: / / affect.media.mit.edu/publications . php

16. Piwek, P., Hernault, H., Prendinger, H., Ishizuka, M.: T2D: Generating dialogues between virtual agents automatically from text. In: Pelachaud, C., Martin, J.-C., André, E., Chollet, G., Karpouzis, K., Pelé, D. (eds.) IVA 2007. LNCS, vol. 4722, pp. 161-174. Springer, Heidelberg (2007)

17. Poggi, I., Pelachaud, C., de Rosis, F.: Eye Communication in a Conversational 3D Synthetic Agent. The European Journal on Artificial Intelligence 13(3), 169-182 (2000)

18. Ran, L., Helal, A., Moore, S.E., Ramachandran, B.: Drishti: An Integrated Indoor/Outdoor Blind Navigation System and Service. In: Proc. IEEE PERCOM 2004, pp. 23-30 (2004)

19. Roden, T.E., Parberry, I., Ducrest, D.: Toward mobile entertainment: A paradigm for narrative-based audio only games. Science of Computer Programming 67(1), 76-90 (2007)

20. Ruttkay, Z., Dormann, C., Noot, H.: Evaluating ECAs. What and How? In: Workshop on Embodied Conversational Agents. Let's specify and evaluate them, at AMAAS (2002)

21. van Mulken, S., André, E., Müller, J.: An empirical study on the trustworthiness of lifelike interface agents. In: Proc. HCI International 1999, LEA, vol. 2, pp. 152-156 (1999)

22. Ververidis, D., Kotropoulos, C.: Emotional speech recognition: Resources, features, and methods. Speech Communication 48(9), 1162-1181 (2006)

23. Wilensky, R., Chin, D.N., Luria, M., Martin, J., Mayfield, J., Wu, D.: The Berkeley UNIX Consultant Project. Artificial Intelligence Review 14(1-2), 43-88 (2000)

24. Zajicek, M., Jonsson, I.-M.: Evaluation and context for in-car speech systems for older adults. In: Proc. ACM Latin American Conf. on HCI, pp. 31-39. ACM Press, New York (2005) 